\title{
Biomechanical implications of the congenital bicuspid aortic valve: A finite element study of aortic root function from in vivo data
}

\author{
Carlo A. Conti, MD, ${ }^{a}$ Alessandro Della Corte, $\mathrm{MD}, \mathrm{PhD},{ }^{\mathrm{b}}$ Emiliano Votta, $\mathrm{PhD},{ }^{\mathrm{a}}$ Luca Del Viscovo, $\mathrm{MD},{ }^{\mathrm{c}}$ \\ Ciro Bancone, MD, ${ }^{\mathrm{b}}$ Luca S. De Santo, MD, ${ }^{\mathrm{b}}$ and Alberto Redaelli, $\mathrm{PhD}^{\mathrm{a}}$
}

\begin{abstract}
Objective: Congenital bicuspid aortic valves frequently cause aortic stenosis or regurgitation. Improved understanding of valve and root biomechanics is needed to achieve advancements in surgical repair techniques. By using imaging-derived data, finite element models were developed to quantify aortic valve and root biomechanical alterations associated with bicuspid geometry.
\end{abstract}

\begin{abstract}
Methods: A dynamic 3-dimensional finite element model of the aortic root with a bicuspid aortic valve (type 1 right/left) was developed. The model's geometry was based on measurements from 2-dimensional magnetic resonance images acquired in 8 normotensive and otherwise healthy subjects with echocardiographically normal function of their bicuspid aortic valves. Numeric results were compared with those obtained from our previous model representing the normal root with a tricuspid aortic valve. The effects of raphe thickening on valve kinematics and stresses were also evaluated.
\end{abstract}

Results: During systole, the bicuspid valve opened asymmetrically compared with the normal valve, resulting in an elliptic shape of its orifice. During diastole, the conjoint cusp occluded a larger proportion of the valve orifice and leaflet bending was altered, although competence was preserved. The bicuspid model presented higher stresses compared with the tricuspid model, particularly in the central basal region of the conjoint cusp $(+800 \%)$. The presence of a raphe partially reduced stress in this region but increased stress in the other cusp.

Conclusions: Aortic valve function is altered in clinically normally functioning bicuspid aortic valves. Bicuspid geometry per se entails abnormal leaflet stress. The stress location suggests that leaflet stress may play a role in tissue remodeling at the raphe region and in early leaflet degeneration. (J Thorac Cardiovasc Surg 2010;140:890-6)

Supplemental material is available online.

The aortic root is the anatomic and functional unit that constitutes the proximal end of the aorta and includes the aortic valve, interleaflet triangles, Valsalva sinuses, and sinotubular junction. The bicuspid aortic valve (BAV), the most frequent congenital cardiac malformation, present in approximately $1 \%$ to $2 \%$ of live births, ${ }^{1,2}$ is thought to result from the intrauterine fusion or nonseparation of 2 underdeveloped cusps, the first mechanism being more likely than the second mechanism. ${ }^{2}$ The right and left coronary leaflets or, less frequently, the right coronary and noncoronary leaflets

From the Department of Bioengineering, Politecnico di Milano, ${ }^{a}$ Milan, Italy; Depart-

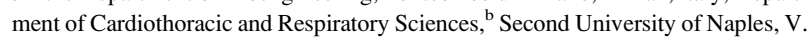
Monaldi Hospital, Naples, Italy; and Department of Experimental and Clinical Internal Medicine, ${ }^{\mathrm{c}}$ Second University of Naples, Naples, Italy.

This study was supported by a grant (PRIN co-funding 2006; prot 2006063487) from the Italian Ministry for University and Research.

Disclosures: None.

Received for publication Nov 13, 2009; revisions received Dec 30, 2009; accepted for publication Jan 10, 2010; available ahead of print April 5, 2010.

Address for reprints: Alessandro Della Corte, MD, PhD, Via P Neruda 6, 81031 Aversa-CE, Italy (E-mail: aledellacorte@libero.it).

0022-5223/\$36.00

Copyright (c) 2010 by The American Association for Thoracic Surgery doi:10.1016/j.jtcvs.2010.01.016 are usually fused. In most cases, the fusion is characterized by a raphe, a region of fibrous thickening at the seam between the 2 fused leaflets.

BAV is widely recognized as a frequent cause of aortic stenosis and aortic regurgitation ${ }^{1-3}$ and is a risk factor for the development of aortic aneurysms. Ascending aortic aneurysm develops in $50 \%$ to $70 \%$ of patients with BAV. ${ }^{1,2,4}$ The still unidentified genetic defect causing BAV malformation is thought to be responsible for the occurrence of these complications; however, some authors ${ }^{3}$ have emphasized the role of altered biomechanics and hemodynamics in the early failure of valve function.

To date, BAV-related phenomena have been studied mainly by means of clinical studies ${ }^{4}$ and histologic or molecular analyses. ${ }^{5,6}$ However, in such studies, it is not possible to discriminate the effects of the genetic defects from those of the biomechanical determinants, because both factors may cooperate to produce the observed disease phenotype.

A computational analysis based on finite element modeling and focused purely on the biomechanical aspects would complete the already available information by quantifying the possible abnormal stresses and strains associated with BAV. This approach has been used by different research groups to analyze normal aortic function ${ }^{7,8}$ or the effect of different valve-sparing procedures. ${ }^{9}$ In recent years, repair techniques for dysfunctional BAV have received increasing 


\section{Abbreviations and Acronyms \\ $\mathrm{BAV}=$ bicuspid aortic valve \\ MRI = magnetic resonance imaging \\ $\mathrm{TAV}=$ tricuspid aortic valve}

attention, highlighting the need for an improved understanding of the functional anatomy of the bicuspid valve-root unit; ${ }^{10-12}$ however, to our knowledge only 2 studies have used a computational approach to address the BAV question, ${ }^{3,13}$ both with some methodological limitations. Robicsek and coworkers ${ }^{3}$ studied the altered hemodynamics downstream of a paradigmatic highly eccentric BAV through a computational fluid-dynamics model, where solid tissues are assumed to be perfectly rigid. Weinberg and Kaazempur Mofrad $^{13}$ developed a fluid structure interaction model, characterized by a sophisticated multiscale constitutive model of tissue stress-strain response: however, to limit the high inherent computational cost, they assumed the aortic root as a symmetric structure, consisting of 2 identical leaflet-sinus units, and analyzed only a quarter of the modeled system. Real BAV root morphology is far more complex.

The present work introduces a novel dynamic structural finite element model that simulates the function of the aortic root with BAV throughout the cardiac cycle. The model combines a realistic 3-dimensional geometry, based on quantitative magnetic resonance imaging (MRI) data, with a constitutive model of leaflet tissue that better describes its complex mechanical response. The model was used to assess whether BAV-related geometric alterations may induce abnormal stresses possibly involved in the occurrence of degenerative phenomena and to analyze the impact of raphe development on valvular function and stresses.

\section{MATERIALS AND METHODS}

\section{Aortic Root Morphologic Characterization}

Aortic root geometry was characterized by the same procedure described in our previous study on the tricuspid aortic valve (TAV). ${ }^{14}$ In brief, bidimensional T1-weighted MRI scans of the heart in the 4-chamber view, the left ventricular output tract, the ascending aorta, and the aortic arch were acquired in 8 normotensive and otherwise healthy subjects with BAV ( 7 men and 1 woman with a mean age of $34 \pm 12$ years) with a body surface of $1.91 \pm 0.15 \mathrm{~m}^{2}$ (mean \pm standard deviation). All subjects were selected on the basis of transthoracic echocardiography showing fusion between the right and left coronary leaflets (BAV type 1, right/left ${ }^{15}$ ) with no stenosis or regurgitation. The study was approved by the institutional review boards, and all subjects provided written informed consent before undergoing MRI. MRI sequence parameters were the same as previously reported. ${ }^{14}$

In the end-diastolic frame, aortic root main geometric parameters were measured (Table 1), including the annulus diameter, commissural positions, width and height of the Valsalva sinuses, and ascending aorta orientation. Each measurement was averaged from 3 off-line estimations by 2 blinded experienced operators. The intercommissural distances (ie, distances be- tween the distal apexes of the 3 interleaflet triangles) were measured in short-axis views, in the transverse plane nearest to the annulus where the commissures were still visible, an average of $4.8 \pm 0.9 \mathrm{~mm}$ above the annular level. Three Valsalva sinuses were detected in all subjects. The width and height of the sinuses were measured in the 3 longitudinal planes passing through each commissure and the midpoint of the opposite sinus (Figure E1). A smaller interleaflet triangle underlying the fusion region of the conjoint cusp was observed, which is typical in BAV. ${ }^{11}$ To better characterize the kinematics of the conjoint cusp, in the systolic frame corresponding to maximal valve opening, the distance between its free margin and the aortic sinus wall in short-axis view and the angle between the leaflet belly and the left ventricle outflow tract in the long-axis view were measured.

The orientation of the ascending aorta was identified by measuring its tilt angle $(\theta)$ with respect to the root in long-axis images and its twist angle $(\varphi)$, formed by the distal ascending aorta and the sagittal plane passing through the midpoint of the right coronary sinus, in short-axis images. Mean values of $\theta$ and $\varphi$ were equal to $6.7 \pm 4.1$ degrees and $25.4 \pm 4.1$ degrees, respectively.

\section{Geometric Modeling and Discretization}

MRI data were used to define the valve orifice area, length of leaflets insertions, profile of the Valsalva sinuses, and position of the ascending aorta The method proposed in our previous study ${ }^{14}$ was properly modified to remove the commissure between the right and left coronary leaflets, reconstructing the conjoint cusp, and to reproduce the abnormal configuration of the interleaflet triangles. Consistent with MRI observations, the height of the triangle underlying the fusion region was $50 \%$ shorter than observed in the other triangles.

With regards to leaflet thickness distribution, 2 different models were developed and termed "model NT" (no thickening) and "model T" (thickening of the raphe). In the first model, the same distribution adopted by Grande and colleagues ${ }^{7}$ and by our TAV model ${ }^{14}$ was used. In the second model, the raphe region was assumed 4 times thicker than the leaflet belly.

Because the adopted MRI data were obtained at end diastole, the unloaded geometric model of the aortic root was defined through a procedure aimed at obtaining consistency with MRI data once the modeled root was loaded with $80 \mathrm{~mm} \mathrm{Hg}$ aortic and ventricular pressures, which normally characterize this time-point of the cardiac cycle. ${ }^{14}$ The geometric model (Figure E2) was discretized with 37001 3-node shell elements (ABAQUS type S3R).

\section{Tissues Physical Properties}

The tissue of the aortic leaflets might be described as fiber-reinforced material, the constituents of which (collagen and elastin) determine the biomechanical behavior of the leaflets' tissue; collagen fibers, crimped when the tissue is unloaded, are preferentially oriented parallel to the annulus. ${ }^{16}$ Thus, the leaflets' mechanical response is nonlinear because of the progressive uncoiling of collagen fibers during tissue tensile loading, and transversely isotropic, because of the preferential orientation of the fibers. This behavior was modeled by a strain energy function $\mathrm{W}:{ }^{17}$

$$
\mathrm{w}=\mathrm{c}_{0}\left(\exp \left(\mathrm{c}_{1}\left(\mathrm{I}_{1}-3\right)^{2}+\mathrm{c}_{2}\left(\mathrm{I}_{4}-1\right)^{2}\right)-1\right)
$$

where $\mathrm{I}_{1}=\operatorname{tr}(\mathrm{C})$ and $\mathrm{I}_{4}=\mathrm{a}_{0} \cdot \mathrm{C} \cdot \mathrm{a}_{0}=\lambda^{2} ; \mathrm{a}_{0}$ is the unit vector that defines the preferential direction of the fibers in the material in the undeformed shape and $\lambda$ is the stretch of the fibers and $\mathrm{C}=\mathrm{F} \cdot \mathrm{F}^{\mathrm{T}}$ is the right Cauchy-Green deformation tensor. Because $\mathrm{F}$ is the deformation gradient tensor, defined as $\mathrm{F}=\partial \mathrm{x} / \partial \mathrm{X}$, that is, the derivative of the current positions as regards to the undeformed position, the Cauchy-Green tensor physically gives the square of local change in distances caused by deformation. In W, the term $\left(I_{1}-3\right)^{2}$ is connected to the isotropic response of the elastin matrix, whereas the term $\left(I_{4}-1\right)^{2}$ is linked to the collagen fibers response and is activated only if $\mathrm{I}_{4}$ is 1 or more. The incompressibility condition implies that $\mathrm{J}=\operatorname{det} \mathrm{F}=1$. 
TABLE 1. Magnetic resonance imaging measurements of the bicuspid aortic valve root in the end-diastolic frame

\begin{tabular}{lrrr}
\hline & \multicolumn{1}{c}{$\begin{array}{c}\text { Right } \\
\text { coronary }\end{array}$} & $\begin{array}{c}\text { Left } \\
\text { coronary }\end{array}$ & Non-coronary \\
\hline Intercommisural distance & $21.8 \pm 4.0$ & $20.6 \pm 2.9$ & $25.3 \pm 3.3$ \\
Annular diameter & $23.6 \pm 2.9$ & $23.2 \pm 3.1$ & $25.2 \pm 4.1$ \\
Intermediate width & $29.4 \pm 3.8$ & $29.4 \pm 3.6$ & $31.7 \pm 4.3$ \\
Height of intermediate width & $4.5 \pm 1.2$ & $4.6 \pm 0.7$ & $4.6 \pm 0.6$ \\
Maximum width & $32.6 \pm 4.2$ & $32.1 \pm 4.5$ & $34.1 \pm 4.4$ \\
Height of maximum width & $9.0 \pm 1.9$ & $9.1 \pm 1.3$ & $9.2 \pm 1.3$ \\
STJ diameter & $27.1 \pm 4.7$ & $27.6 \pm 5.2$ & $29.7 \pm 5.2$ \\
Height of the sinuses & $19.4 \pm 2.7$ & $18.4 \pm 1.8$ & $21.2 \pm 3.6$ \\
\hline
\end{tabular}

Values are expressed in millimeters (mean \pm standard deviation). Right coronary, left coronary, and noncoronary refer to the 3 longitudinal views in which the parameters were measured (see text). STJ, Sinotubular junction.

The same constitutive parameters used for the TAV model ${ }^{14}$ were adopted for the BAV model. They were set equal to $\mathrm{c}_{0}=4.8 \mathrm{kPa}, \mathrm{c}_{1}=0.26$, $\mathrm{c}_{2}=3.83$ by fitting equation (1) to the model reported by Billiar and Sacks. ${ }^{16}$ Equation (1) was implemented into the ABAQUS/Explicit code with an external subroutine.

The mechanical response of the other tissues was assumed linear, elastic, and isotropic, with a 2 MPa Young modulus and a 0.3 Poisson ratio. ${ }^{8}$ A density of $1.1 \mathrm{~g} / \mathrm{cm}^{3}$ was assumed for all tissues. ${ }^{8}$

\section{Boundary Conditions and Interactions}

A 2-step dynamic simulation was performed on the model. First, $80 \mathrm{~mm}$ $\mathrm{Hg}$ of pressure was applied to the model, thus obtaining end-diastolic loading conditions at which the model's dimensions are consistent with enddiastolic MRI data. Second, physiologic time-dependent pressures were applied to aortic root substructures. As in previous studies, ${ }^{8,9,14}$ the leaflets were assumed to be approximately stress-free in the open position. Because the valve in vivo is not yet open at end diastole (assumed as simulation starting point), 2 cardiac cycles were simulated, the second cycle having a more realistic initial configuration and accounting for inertial effects caused by previous motion.

Proper nodal displacements were imposed to the nodes belonging to the annulus to mimic ventricular contraction, consistent with data reported by Lansac and colleagues. ${ }^{18}$ A general contact algorithm and a friction coefficient equal to 0.05 were assumed to describe the leaflets' interaction tangentially to their contact surface when coapting. ${ }^{19}$ The ABAQUS/Explicit software version 6.7-1 (ABAQUS/Explicit; SIMULIA Inc, Providence $\mathrm{RI})$ was used to perform the numeric analysis.

\section{Biomechanical Analysis}

Aortic root biomechanics during the cardiac cycle were analyzed in terms of valve opening and closure timing and leaflets coaptation. Moreover, stresses acting on aortic root components were computed. The effect of the mere alteration of geometric proportions associated with leaflets fusion was assessed by comparing the results from the NT model with those from our previous physiologic TAV model. ${ }^{14}$ The implications of raphe thickening were analyzed by comparing the NT and T models.

\section{RESULTS}

\section{Valve Kinematics and Coaptation}

Radial displacements of the noduli of Arantius computed with the NT model showed a reduced motility of the conjoint cusp compared with the physiologic displacements (Figure 1, $A$ ), resulting in an eccentric elliptic orifice during systole (Figure 1,B), in agreement with MRI data (Figure 1,C)
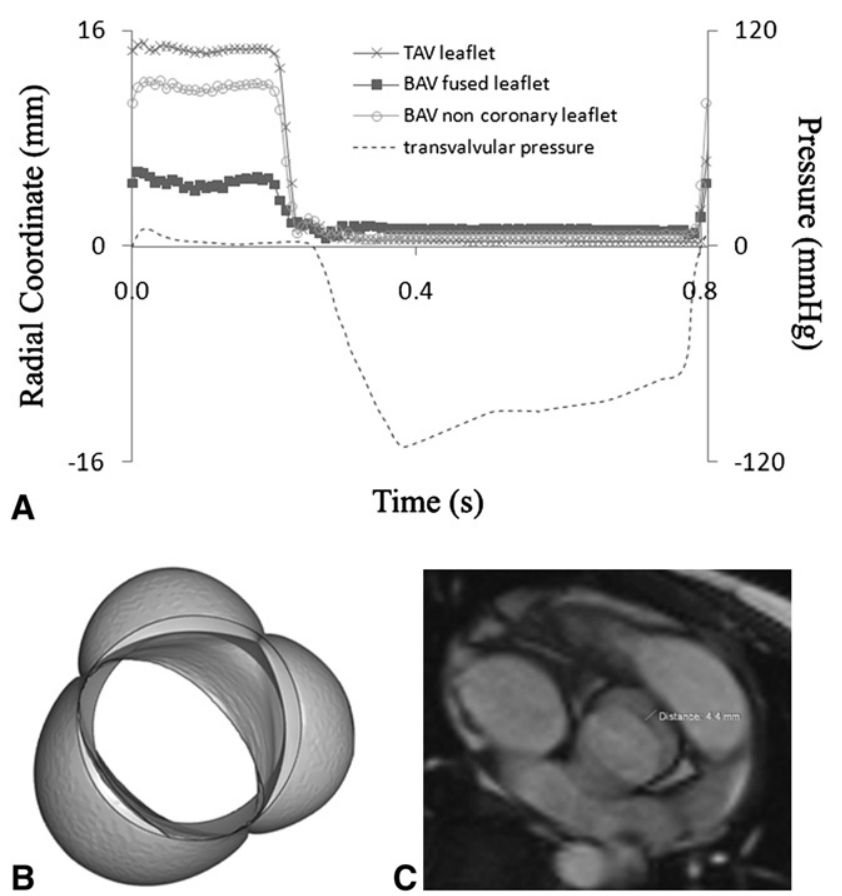

FIGURE 1. Comparison between BAV and TAV kinematics. A, Radial displacements of the noduli of Arantius of TAV leaflets (averaged value), BAV conjoint cusp, and BAV noncoronary leaflet plotted with the transvalvular pressure. B, Aortic view of the BAV model at maximal systolic opening. C, Short-axis view MRI scan at the maximal systolic opening frame. $B A V$, Bicuspid aortic valve; $T A V$, tricuspid aortic valve.

and experimental tests. ${ }^{3}$ No noticeable difference in opening and closure timing was observed when compared with the TAV model.

During diastole, the conjoint cusp occluded a larger proportion of the valve orifice $(64 \%)$ and leaflet bending was altered. Compared with leaflets coaptation in the TAV model, the noduli of Arantius were closer to the annular plane, with a $36 \%$ decrease in coaptation height, and contact pressure was reduced by $41 \%$. However, consistent with MRI observations, valve competence was preserved.

\section{Leaflet Stresses}

The NT and TAV models ${ }^{14}$ are compared in Figure 2, in terms of distribution of leaflet in-plane maximum principal stresses at different pressure loads during the cardiac cycle. Peak values, corresponding to the peak transvalvular pressure decrease $(108 \mathrm{~mm} \mathrm{Hg})$, are summarized in Table E1 for 4 different leaflet regions: the free margin zone, coaptation area, belly region, and attachment edge.

In the NT model, stresses were markedly lower in the noncoronary cusp than in the conjoint cusp. The difference was notable in all regions: $-34 \%$ in the free margin zone, $-47 \%$ in the coaptation area, $-52 \%$ in the belly region, and $-94 \%$ in the attachment edge. Compared with normal leaflets (TAV model), the noncoronary leaflet in the NT model had a similar 

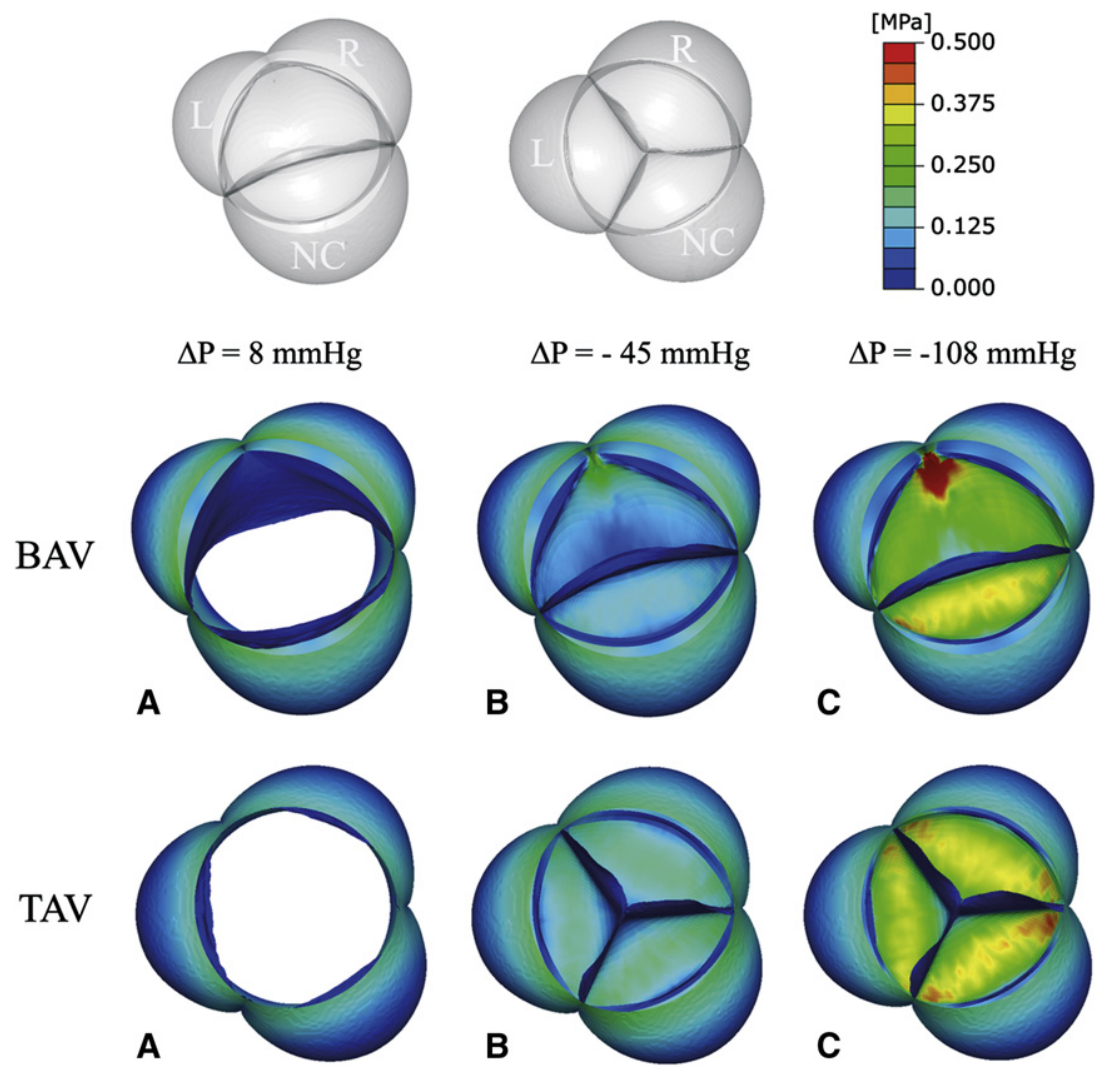

FIGURE 2. Maximum principal stress distributions on the aortic root throughout the cardiac cycle for the BAV (top) and TAV (bottom) models. BAV, Bicuspid aortic valve; $T A V$, tricuspid aortic valve; $R$, right coronary sinus; $L$, left coronary sinus; $N C$, noncoronary sinus.

peak stress distribution, although characterized by lower values. These were reduced by $19 \%$ in the coaptation area, $49 \%$ in the belly region, and $30 \%$ in the attachment edge. Instead, the conjoint leaflet experienced higher peak stresses. These were increased by $0.5 \%$ in the coaptation area, $33 \%$ in the belly region, and $800 \%$ in the attachment edge.

\section{Aortic Root Stresses}

For each Valsalva sinus in the NT model, circumferential stresses at peak aortic pressure were highest next to the sinotubular junction, whereas longitudinal stresses were highest next to the commissures. This pattern was comparable to that computed with the TAV model. Circumferential stresses were maximal $(234 \mathrm{kPa})$ in the smallest sinus (ie, the left sinus), and longitudinal stresses were maximal (148 kPa) in the largest sinus, that is, the noncoronary sinus in the BAV model and the right sinus in the TAV model (Table E2). In regard to the proximal part of the ascending aorta, circumferential and longitudinal stresses reached values of 225 and $64 \mathrm{kPa}$ at the inner curvature ("concavity") and 216 and $86 \mathrm{kPa}$ at the outer curve ("convexity"), respectively. These corresponded to $8 \%$ and $20 \%$ increases at the concavity and $17 \%$ and $36 \%$ increases at the convexity, respectively, when compared with the TAV model
(Figure 3). The longitudinal to circumferential stress ratio was thereby increased by $16 \%$ at the convexity.

\section{Influence of Raphe Thickening on Leaflet Kinematics and Stress Distribution}

The thickening of the raphe region in the T model affected the shape of the conjoint cusp during closure and diastole. Its 2 halves bulged slightly more toward the ventricle than the raphe region, but coaptation was preserved.

Leaflet in-plane maximum principal stresses at peak transvalvular pressure load are displayed in Figure 4 for the NT and $\mathrm{T}$ models. The thickening of the raphe region reduced the peak stresses in the central portion of its attachment edge region by an average of $55 \%$ compared with the corresponding values in the NT model. The belly of the noncoronary leaflet was slightly more stressed than in the NT model, with a $5 \%$ increase in maximum in-plane principal stresses.

\section{DISCUSSION}

On the basis of MRI-derived morphologic parameters from 8 patients, a dynamic 3-dimensional finite element model of the aortic root affected by BAV malformation was developed. The model allowed for the assessment of the biomechanical implications of BAV presence in the early 

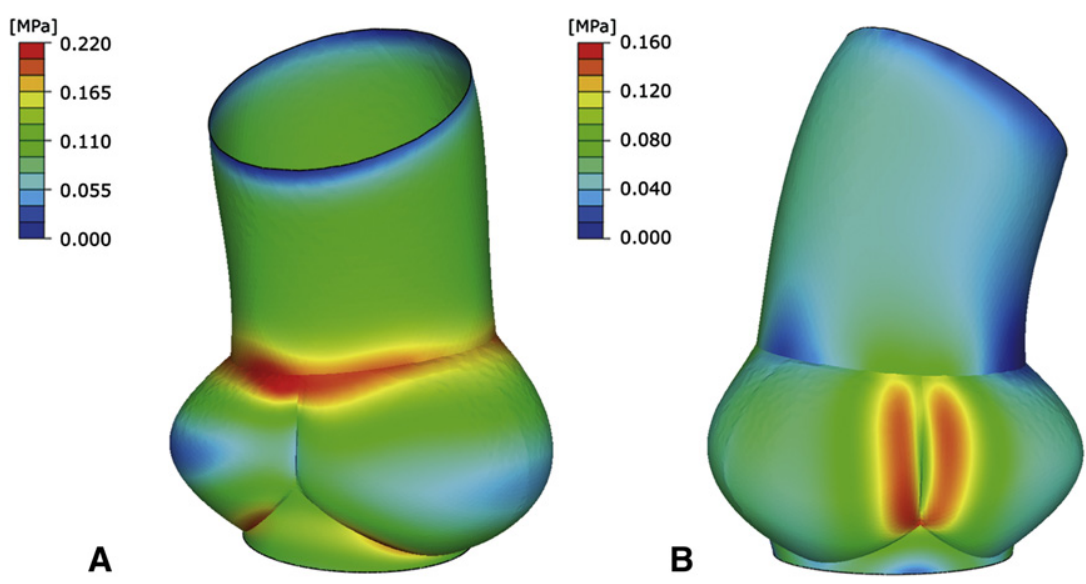

\section{C}

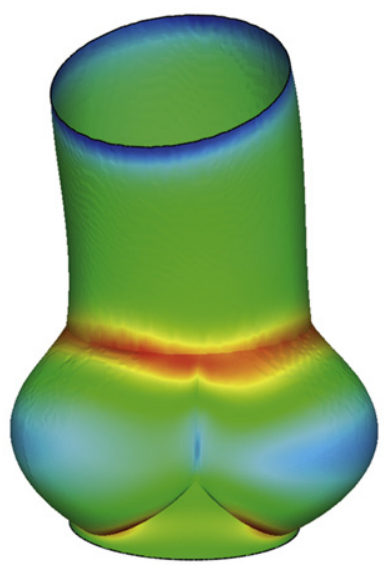

D

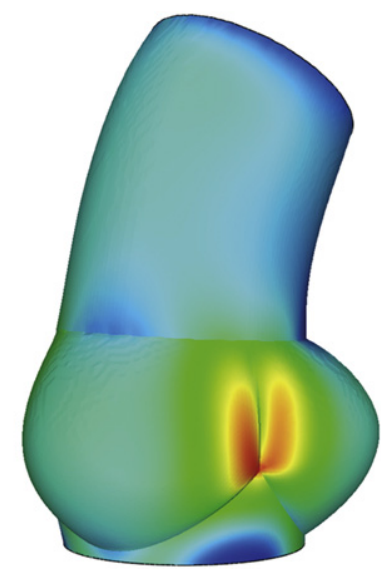

FIGURE 3. Maximum principal stress distribution on the aortic root at peak aortic pressure: circumferential stresses on BAV (A) and TAV (C); longitudinal stresses on BAV (B) and TAV (D).

stage of the disease, before valve or aorta complications have occurred. It is now available for possible modifications to simulate both complications and repair.

The MRI measurements seemed to be reliable, given the high intra- and inter-operator repeatability and the consistency with previous findings from the literature (eg, the conjoint cusp covered an area slightly smaller than expected for 2 normal cusps, whereas the noncoronary cusp was slightly larger, complying with the classic ex vivo descriptions). ${ }^{2}$

\section{Bicuspid Aortic Valve Kinematics}

Morphologic anomalies characterizing the aortic root with BAV led to 2 major differences in valve kinematics compared with TAV. First, during systole the BAV did not open as widely and centrally as TAV, resulting in an
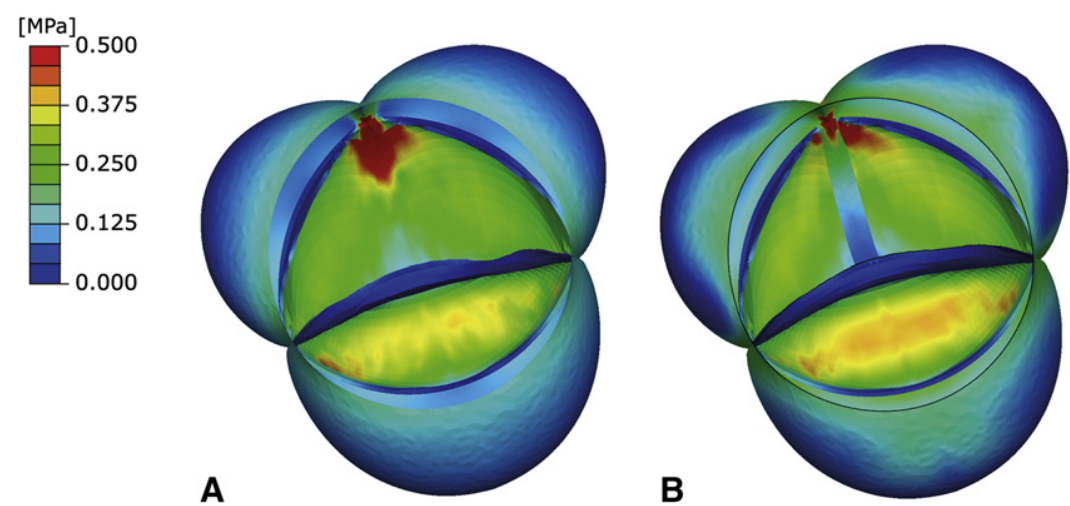

FIGURE 4. Maximum principal stress distributions on the aortic leaflets for 2 different models of the conjoint cusp. A, NT model without raphe. B, T model with raphe (ie, a thicker region at the site of cusp fusion). 
elliptic shape of the orifice. This behavior was observed in both the T and NT models, indicating that the mere fusion of the 2 coronary leaflets, regardless of raphe presence or absence, restricts the opening of the conjoint cusp, causing subclinical stenosis. Other authors have reported that the geometric differences between effective opening area of stenotic BAVs and TAVs are responsible for echocardiography underestimating the degree of stenosis in patients with BAV. If valve orifice areas are equal, a more eccentric opening yields greater flow acceleration and thereby a more severe functional stenosis. ${ }^{20}$ Notably, because only subjects with BAV with no stenosis at echo-Doppler evaluation were included in the present study, our findings may extend the above concept, indicating that the normal echocardiographic function of a BAV corresponds to some degree of stenosis. This could lead to reconsidering the notion of post-stenotic dilatation of the ascending aorta in the BAV setting, supporting the hypothesis that even the "normal" BAV may cause enough flow alterations to drive aortic wall remodeling processes. ${ }^{3}$

The second important finding was that coaptation did not occur in the midline during diastole. The line of cusp contact was laterally displaced with the conjoint cusp occluding a larger proportion of the valve orifice. This is a compensatory mechanism that preserves coaptation but implies an increased bulge of the leaflet bellies. Both cusps undergo more flexure compared with the normal tricuspid valve and are tightly stretched to maintain coaptation. These numeric data agree with experimental observations. ${ }^{3}$ Coaptation height is argued to be an important parameter, possibly capable of predicting postprocedural prognosis after aortic valve repair. ${ }^{12}$ Although we modeled only the "normal" condition, a 36\% reduction in coaptation height was observed in BAV compared with TAV. Because coaptation height is expected to progressively decrease as the root dilates, this evidence may indicate that a lesser degree of aortic root dilatation is required for BAV to become secondarily regurgitant, also explaining previous echocardiographic observations. $^{21}$

\section{Stress Magnitude and Location}

As in the TAV model, ${ }^{14}$ in both the NT and T models peak stresses in diastole were located in the leaflet belly for the noncoronary cusp, but abnormal peak stresses were detected in the attachment edge of the conjoint cusp for the maximum transvalvular pressure decrease, and its belly region presented the highest stresses concentrated in the central basal area. This abnormal peak stress may represent the pathogenetic link between 2 phenomena that characterize BAV: the presence of an abnormal interleaflet triangle and the formation of raphe. The morphology of the crown-like structure formed by leaflet attachments to the root wall is disarrayed in $\mathrm{BAV}$, because the interleaflet triangle below the rudimentary commissure corresponding to the fusion site is smaller than the other 2 triangles,${ }^{11}$ causing a nonphysiologic creasing of the conjoint cusp. The increased stress at the fusion site in turn may be the stimulus for a partly compensatory reactive process, that is, fibrotic remodeling eventually complicated by calcification. ${ }^{22}$ Indeed, the presence of a thickened region at the fusion site reduced overall stress on the conjoint cusp, however, entailing an increase in stress at the belly region of the nonfused leaflet. This hypothesis is in accordance with the observation that the raphe seems to preferentially develop in BAVs with unequal cusp sizes, ${ }^{23}$ such as those we selected for MRI measurements, rather than in those with 2 equally sized cusps, such as in Weinberg and Kaazempur Mofrad's ${ }^{13}$ model.

The BAV morphology involved abnormal stresses, which suggests that surgical repair procedures should aim to restore competence of a regurgitant BAV as well as to "tricuspidalize" it, because our data show that the conjoint cusp, although normally coapting, and even after resection of the fusion region (NT model), is overloaded with stress and may therefore be prone to accelerated degeneration or tearing.

Morphologic anomalies associated with BAV lead to increased stresses in the leaflets and surrounding structures. In particular, longitudinal stresses were increased by $36 \%$, compared with the TAV model, at the greater curvature of the ascending aorta. This stress location corresponds to the region (usually referred to as the "convexity" of the ascending aortic profile ${ }^{6,24}$ ) where BAV-related dilatation is more pronounced. ${ }^{24}$ According to our previous studies, greater degrees of extracellular matrix disarray and smooth muscle cell changes at the convexity wall compared with the contralateral wall underlie these asymmetric dilatations, ${ }^{6}$ suggesting that the hypothetic culprit gene causing BAV-related aneurysms, if any, should be explored among those regulating arterial wall response to the mechanical stimulus. The "convexity" is also the site where aortic dissection originates most frequently. ${ }^{25}$ The increased longitudinal to circumferential stress ratio is consistent with the fact that the intimal tear is usually circumferential.

\section{Study Limitations}

Despite the consistency of numeric results with experimental and clinical evidence from the literature, the model may still be improved, either to remove some current limitations or to extend its use to the analysis of other scenarios, for example, simulating the later stages of BAV disease, the mechanisms responsible for regurgitation, and even their respective surgical corrections.

To this purpose, possible improvements include the modeling of the anisotropic and nonlinear response of the Valsalva sinuses and ascending aorta. Furthermore, the mechanical response implemented for the BAV model was the same as adopted in the TAV model. As previously mentioned, we focused on the effects of the mere morphology, 
neglecting possible variability of the tissue properties between the 2 scenarios, especially at the aortic level. ${ }^{25}$ Also, the effects of flow dynamics were not considered in our purely structural model.

In the T model, the raphe region was modeled by thickening the central zone of the conjoint cusp, not accounting for a detailed description of the raphe 3-dimensional morphology. This choice was made because of the unreliability of MRI in raphe visualization and measurement, and because available data from the literature typically refer to explanted highly stenotic and calcified BAVs. ${ }^{22,23}$

\section{CONCLUSIONS}

The present study demonstrated that biomechanical abnormalities affect the BAV even when defined as normally functioning by echocardiography. Both the valve kinematics and the spatial pattern of leaflet stress distribution suggest that raphe development and early occurrence of regurgitation or stenosis may strictly depend on those abnormalities. Moreover, the mere BAV anatomy, at least in its most frequent form (type $1 \mathrm{~L} / \mathrm{R}$ ), regardless of the possible coexistence of some inherited aortic wall weakness, implies altered aortic wall stress amount and distribution, which may play a role in determining the unique form of aortopathy that frequently develops with BAV.,

\section{References}

1. Fedak PW, Verma S, David TE, Leask RL, Weisel RD, Butany J. Clinical and pathophysiological implications of a bicuspid aortic valve. Circulation. 2002; 106:900-4.

2. Braverman AC, Güven H, Beardslee MA, Makan M, Kates AM, Moon MR. The bicuspid aortic valve. Curr Probl Cardiol. 2005;30:470-522.

3. Robicsek F, Thubrikar MJ, Cook JW, Fowler B. The congenitally bicuspid aortic valve: how does it function? Why does it fail? Ann Thorac Surg. 2004;77:177-85.

4. Della Corte A, Bancone C, Quarto C, Dialetto G, Covino FE, Scardone M, et al. Predictors of ascending aortic dilatation with bicuspid aortic valve: a wide spectrum of disease expression. Eur J Cardiothorac Surg. 2007;31:397-404.

5. Ikonomidis JS, Jones JA, Barbour JR, Stroud RE, Clark LL, Kaplan BS, et al. Expression of matrix metalloproteinases and endogenous inhibitors within ascending aortic aneurysms of patients with bicuspid or tricuspid aortic valves. $J$ Thorac Cardiovasc Surg. 2007;133:1028-36.

6. Della Corte A, Quarto C, Bancone C, Castaldo C, Di Meglio F, Nurzynska D, et al. Spatiotemporal patterns of smooth muscle cell changes in ascending aortic dilatation with bicuspid and tricuspid aortic valve stenosis: focus on cell-matrix signaling. J Thorac Cardiovasc Surg. 2008;135:8-18.
7. Grande KJ, Cochran RP, Reinhall PG, Kunzelman KS. Stress variations in the human aortic root and valve: the role of anatomic asymmetry. Ann Biomed Eng. 1998;26:534-45.

8. Gnyaneshwar R, Kumar RK, Balakrishnan KR. Dynamic analysis of the aortic valve using a finite element model. Ann Thorac Surg. 2002;73:1122-9.

9. Soncini M, Votta E, Zinicchino S, Burrone V, Mangini A, Lemma M, et al. Aortic root performance after valve sparing procedure: a comparative finite element analysis. Med Eng Phys. 2009;31:234-43.

10. El Khoury G, Vanoverschelde JL, Glineur D, Pierard F, Verhelst RR, Rubay J, et al. Repair of bicuspid aortic valves in patients with aortic regurgitation. Circulation. 2006;114:I610-6.

11. Pretre R, Kadner A, Dave H, Bettex D, Genoni M. Tricuspidisation of the aortic valve with creation of a crown-like annulus is able to restore a normal valve function in bicuspid aortic valves. Eur J Cardiothorac Surg. 2006;29: 1001-6.

12. Lansac E, Di Centa I, Raoux F, Bulman-Fleming N, Ranga A, Abed A, et al. An expansible aortic ring for a physiological approach to conservative aortic valve surgery. J Thorac Cardiovasc Surg. 2009;138:718-24.

13. Weinberg EJ, Kaazempur Mofrad MR. A multiscale computational comparison of the bicuspid and tricuspid aortic valves in relation to calcific aortic stenosis. J Biomech. 2008;41:3482-7.

14. Conti CA, Votta E, Della Corte A, Del Viscovo L, Bancone C, Cotrufo M, et al. Dynamic finite element analysis of the aortic root from MRI-derived parameters. Med Eng Phys. 2010 Jan 7. [Epub ahead of print].

15. Sievers H-H, Schmidtke C. A classification system for the bicuspid aortic valve from 304 surgical specimens. J Thorac Cardiovasc Surg. 2007;133:1226-33.

16. Billiar KL, Sacks MS. Biaxial mechanical properties of the native and glutaraldehyde-treated aortic valve cusp: Part II—a structural constitutive model. J Biomech Eng. 2000;122:327-35.

17. May-Newman K, Yin FC. A constitutive law for mitral valve tissue. J Biomech Eng. 1998;120:38-47.

18. Lansac E, Lim HS, Shomura Y, Lim KH, Rice NT, Goetz W, et al. A fourdimensional study of the aortic root dynamics. Eur J Cardiothorac Surg. 2002; 22:497-503.

19. Votta E, Maisano F, Bolling SF, Alfieri O, Montevecchi FM, Redaelli A. The Geoform disease-specific annuloplasty system: a finite element study. Ann Thorac Surg. 2007;84:92-101.

20. Donal E, Novaro GM, Deserrano D, Popovic ZB, Greenberg NL, Richards KE, et al. Planimetric assessment of anatomic valve area overestimates effective orifice area in bicuspid aortic stenosis. J Am Soc Echocardiogr. 2005;18:1392-8.

21. Alegret JM, Vernis JM, Palazón O, Borràs X, Duran I. Factors related to aortic regurgitation in the presence of a dilated aortic root. Am J Cardiol. 2005;95: 417-20.

22. Otto CM. Calcification of bicuspid aortic valves. Heart. 2002;88:321-2.

23. Sabet HY, Edwards WD, Tazelaar HD, Daly RC. Congenitally bicuspid aortic valves: a surgical pathology study of 542 cases (1991 through 1996) and a literature review of 2,715 additional cases. Mayo Clin Proc. 1999;74:14-26.

24. Bauer M, Gliech V, Siniawski H, Hetzer R. Configuration of the ascending aorta in patients with bicuspid and tricuspid aortic valve disease undergoing aortic valve replacement with or without reduction aortoplasty. J Heart Valve Dis. 2006;15: 594-600.

25. Choudhury N, Bouchot O, Rouleau L, Tremblay D, Cartier R, Butany J, et al. Local mechanical and structural properties of healthy and diseased human ascending aorta tissue. Cardiovasc Pathol. 2009;18:83-91. 

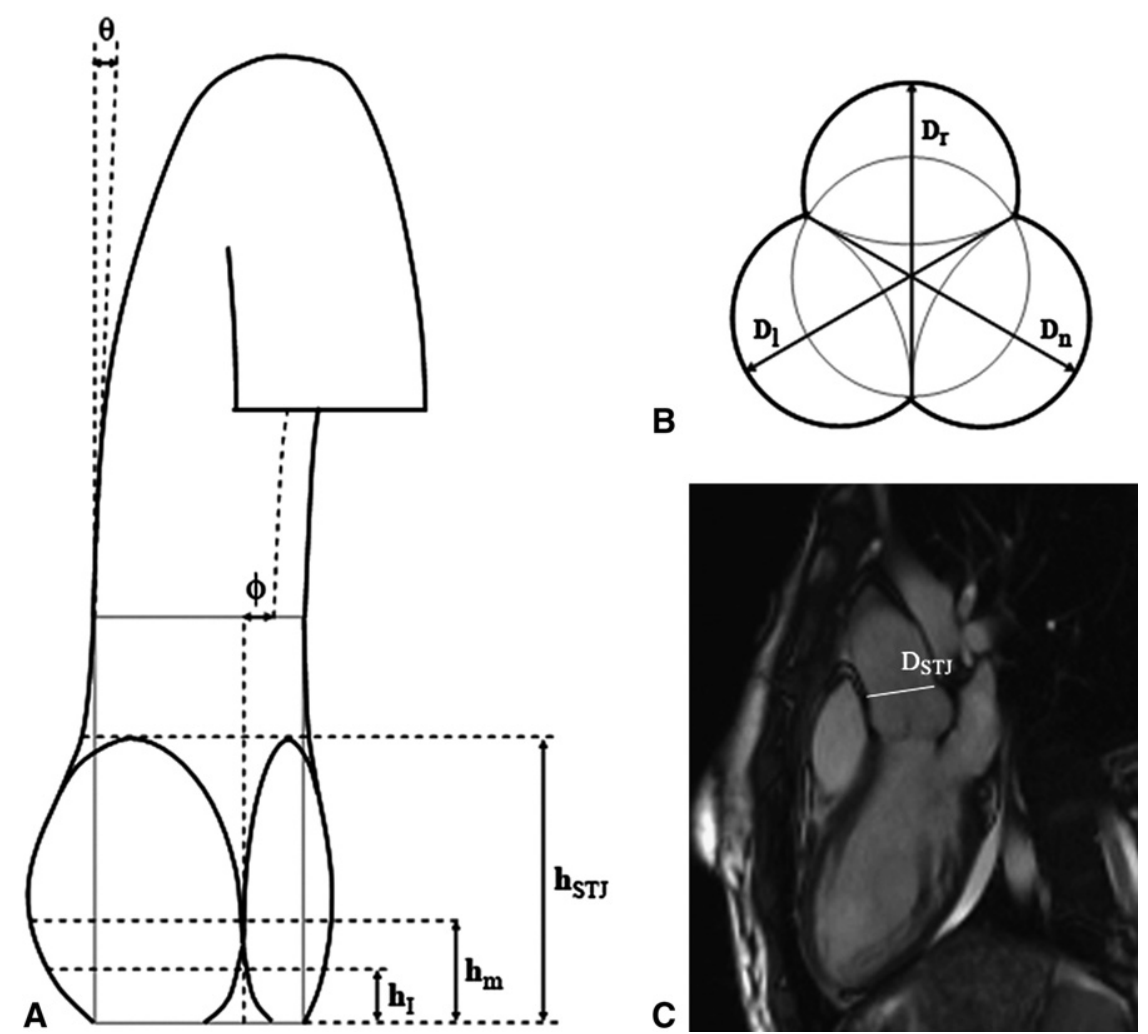

FIGURE E1. Aortic root. A, sinus heights $\left(h_{\mathrm{I}}=\right.$ intermediate level, $\mathrm{h}_{\mathrm{m}}=$ maximum width level, $\mathrm{h}_{\mathrm{STJ}}=$ sinotubular junction level $=$ height of the sinus) and angles of aorta orientation. B, Example of sinus width measurement at the $h_{m}$ level: Lines passing through the point of maximum width of each sinus and its opposite commissure indicate the orientations of the 3 longitudinal views $\left(D_{r}\right.$ for the right coronary, $D_{1}$ for the left coronary, and $D_{n}$ for the noncoronary sinus) where sinus measurements were performed. $C$, Example of a sinotubular diameter measurement $\left(\mathrm{D}_{\mathrm{STJ}}\right)$ on a long-axis view MRI scan oriented in the $\mathrm{D}_{\mathrm{r}}$ plane.
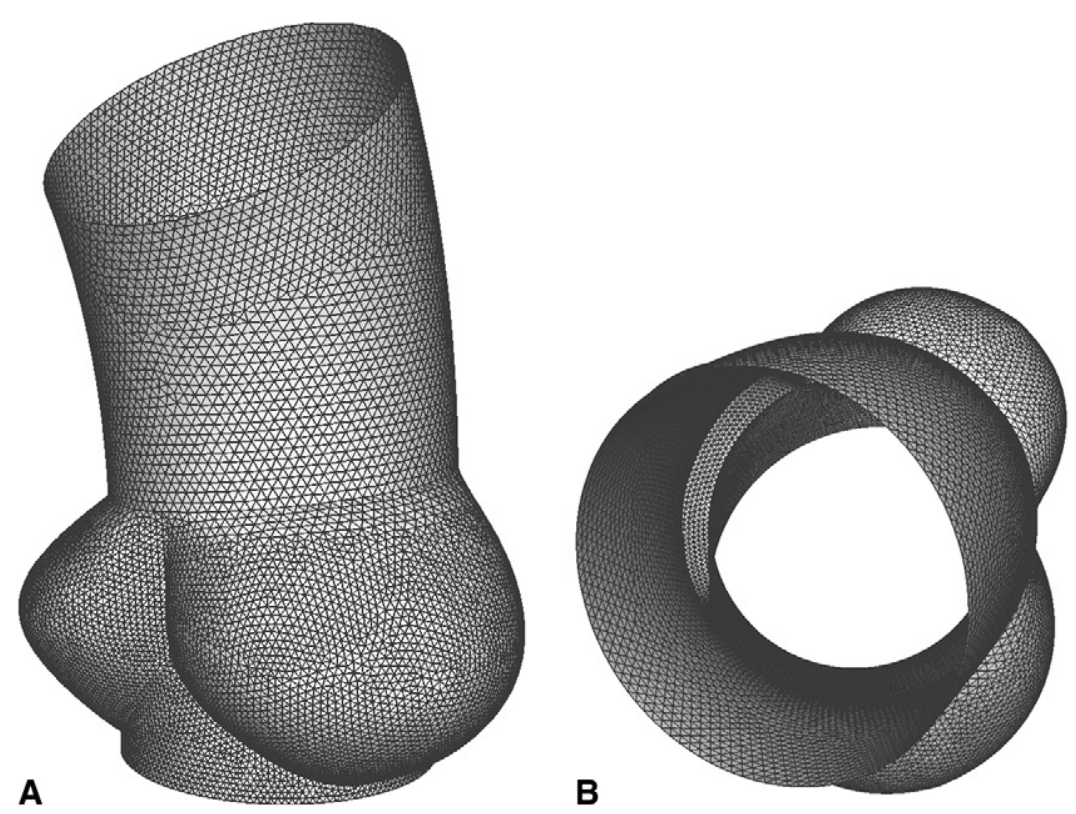

FIGURE E2. Finite element mesh of the aortic root with BAV: frontal (A) and aortic (B) views. 
TABLE E1. Leaflet stresses

\begin{tabular}{lcc}
\hline & Conjoint cusp & Noncoronary cusp \\
\hline FMZ & $13-98$ & 0 to 64 \\
CA & $18-213$ & $0-112$ \\
BR & $22-809$ & $14-388$ \\
AE & -2 to 2929 & -3 to 150 \\
\hline$F M Z$, Free margin zone; $C A$, coaptation area; $B R$, belly region; $A E$, attachment edge. \\
Leaflets in-plane maximum principal stresses at the transvalvular peak ( $t=0.384$ s) are \\
expressed in kilopascals.
\end{tabular}

TABLE E2. Root stresses

\begin{tabular}{lccccc}
\hline & Interleaflet triangles & Left coronary sinus & Right coronary sinus & Noncoronary sinus & Ascending aorta \\
\hline S11 & $81-182$ & $31-234$ & $23-220$ & $40-223$ & $14-225$ \\
S22 & -25 to 106 & $19-138$ & $31-144$ & $19-148$ & $4-86$ \\
\hline S11 & & Circh
\end{tabular}

S11 = Circumferential stresses; S22 = longitudinal stresses. Aortic root in-plane maximum principal stresses at peak aortic pressure $(\mathrm{t}=0.104 \mathrm{~s})$ are expressed in kilopascals. 